\title{
Gene expression analysis in EBV-infected ataxia-telangiectasia cell lines by RNA-sequencing reveals protein synthesis defect and immune abnormalities
}

\author{
Moussab Tatfi ${ }^{1}$, Emeline Perthame ${ }^{4}$, Kenzo-Hugo Hillion ${ }^{4}$, Marie-Agnès Dillies ${ }^{4}$, Hervé Menager ${ }^{4}$, \\ Olivier Hermine ${ }^{1,2,3}$ and Felipe Suarez ${ }^{1,2,3^{*}}$ (1)
}

\begin{abstract}
Background: Epstein-Barr virus (EBV) targets B-cells where it establishes a latent infection. EBV can transform B-cells in vitro and is recognized as an oncogenic virus, especially in the setting of immune compromise. Indeed, immunodeficient patients may fail to control chronic EBV infection, leading to the development EBV-driven lymphoid malignancies. Ataxia telangiectasia (AT) is a primary immune deficiency caused by mutations in the ATM gene, involved in the repair of double-strand breaks. Patients with AT are at high risk of developing cancers, mostly B-cell lymphoid malignancies, most of which being EBV-related. Aside from immune deficiency secondary to AT, loss of ATM function could also hinder the control of the virus within B-cells, favoring lymphomagenesis in AT patients.

Results: We used RNA sequencing on lymphoblastoid cell lines derived from patients with AT and healthy donors to analyze and compare both cellular and viral gene expression. We found numerous deregulated signaling pathways involving transcription, translation, oncogenesis and immune regulation. Specifically, the translational defect was confirmed in vitro, suggesting that the pathogenesis of AT may also involve a ribosomal defect. Concomitant analysis of viral gene expression did not reveal significant differential gene expression, however, analysis of EBV interactome suggests that the viral latency genes EBNA-3A, EBNA-3C and LMP1 may be disrupted in LCL from AT patients.

Conclusion: Our data support the notion that ATM deficiency deregulates cellular gene expression possibly disrupting interactions with EBV latent genes, promoting the oncogenic potential of the virus. These preliminary findings provide a new step towards the understanding of EBV regulation and of AT pathogenesis.
\end{abstract}

Keywords: EBV, ATM, Ataxia, RNA-seq, Lymphoma

\section{Background}

Epstein-Barr virus (EBV) is a human Herpesviridae that infects about $95 \%$ of adults worldwide. Most genes encoded by the viral genome are expressed during the

\footnotetext{
*Correspondence: felipe.suarez@aphp.fr

${ }^{1}$ INSERM U1 163/CNRS ERL8254 - Laboratory of Cellular and Molecular Mechanisms of Hematological Disorders and Therapeutic Implications, Institut Imagine, Paris, France

Full list of author information is available at the end of the article
}

lytic cycle and contribute to the production of viral particles. By contrast, only a restricted repertoire of viral genes is expressed during latency, to allow a lifelong persistence of the virus in the organism. EBV's lytic cycle takes place in the oropharyngeal epithelium whereas the latent cycle is established in the B lymphocytes from the underlying lymphoid tissues [1]. Chronic infection in immunocompetent individuals is generally asymptomatic with the virus being maintained in a latent state [2]. However, inefficient control of viral latency contributes original author(s) and the source, provide a link to the Creative Commons licence, and indicate if changes were made. The images or other third party material in this article are included in the article's Creative Commons licence, unless indicated otherwise in a credit line to the material. If material is not included in the article's Creative Commons licence and your intended use is not permitted by statutory regulation or exceeds the permitted use, you will need to obtain permission directly from the copyright holder. To view a copy of this licence, visit http://creativecommons.org/licenses/by/4.0/. The Creative Commons Public Domain Dedication waiver (http://creativeco mmons.org/publicdomain/zero/1.0/) applies to the data made available in this article, unless otherwise stated in a credit line to the data. 
to the development of malignancies such as Burkitt's lymphoma, Hodgkin's lymphoma and Nasopharyngeal carcinoma.

Several primary immune deficiencies (PID) are associated with poor EBV responses and are also at high risk for EBV-related malignancies [3]. Ataxia telangiectasia (AT) is a rare PID caused by mutations in the Ataxia Telangiectasia Mutated (ATM) gene, involved in the DNA damage response (DDR). AT patients have an increased risk of cancer, mostly B-cell lymphoid malignancies, many of which are related to EBV [4]. The prevailing hypothesis to explain the increased incidence of malignancies in patients with AT is based on the role of the ATM kinase in the DDR [5]. However, the strong association of lymphomas with EBV also suggests an oncogenic role of the latter. AT patients often present with antibody deficiency and T-cell lymphopenia but rarely overt immunodeficiency [6]. A number of PIDs exhibit a selective susceptibility to EBV-related malignancies, while displaying a more restricted susceptibility to other opportunistic infections. In such cases, specific mechanisms may include pathways important for T, NK and iNKT cytotoxicity aimed at EBV-infected B-cells, and pathways involved in expansion of EBV-specific T-cells, leading to an inability to cope with intense EBV induced proliferative stress like in XMEN or CTPS1 mutated patients [7]. We raised the hypothesis that the lack of ATM function in AT patients may be associated with a less stringent control of EBV latency in ATM-deficient B cells, thereby promoting the oncogenic properties of the virus.

Indeed, beside DNA repair, ATM is also involved in a multitude of signaling pathways such as cell cycle checkpoint, apoptosis, mitochondrial metabolism and telomere maintenance [8]. In addition, ATM is involved both in transcription induction [9], and in transcription inhibition in the vicinity of a double-strand break, in nuclear [10] or ribosomal DNA [11]. ATM has a role in the control of the latent cycle of Kaposi's sarcoma herpesvirus (KSHV) and Murine $\gamma$-herpesvirus 68 (MHV68), both related to EBV $[12,13]$.

We performed RNA sequencing (RNA-seq) on lymphoblastoid cell lines (LCL) generated from AT patients and healthy donors, to explore the specific expression pattern of both the cellular and viral genomes and investigate a possible role of ATM in the regulation of EBV latent cycle.

\section{Results}

\section{Identification of differentially expressed genes}

We conducted RNA-seq on LCL from AT patients (LCLAT, $n=7$ ) and healthy donors (LCL-WT, $n=5$ ), previously phenotyped for ATM function (Additional file 1: Fig. S1), using Illumina HiSeq 2500 technology (Fig. 1a).
An average of 82 million paired-end reads were generated per sequenced sample (range 64-138 million). Mapping on the human genome (GRCh38) and the viral genome (V01555.2) gave an average of 63\% (range 56-76\%) and $0.19 \%$ (range $0.10-0.44 \%$ ) of mapped reads respectively (Fig. 1b). A total of 30,794 transcripts were detected using a threshold of one transcript in at least one sample, including 30,687 on the human genome and 107 on the viral genome. Principal component analysis (PCA) shows that LCL-AT and WT segregate into two distinct groups (Fig. 1c).

$1899(6.2 \%)$ of the genes were differentially expressed (adjusted $p$ value $<0.05)$, among which $941(49.5 \%)$ had an absolute $\log 2$ fold-change $(|\log 2 \mathrm{FC}|)>1$. These genes and their corresponding fold-changes are given in Additional file 2: Table S1. A heatmap of the DE genes with $|\log 2 \mathrm{FC}|>1$ demonstrating a separation of the 2 groups is shown in Fig. 1d. Of the 1899 DE genes, 875 were upregulated and 1024 were downregulated in AT (Fig. 1e).

\section{Analysis of the cellular genome}

We applied the over-representation tool from the PANTHER classification system to the list of DE genes. 1703 (90\%) DE genes were annotated in the GO-database [14]. Over-represented categories, which are more highly represented in the DE gene list than would be expected by chance [15], were determined for biological process, molecular function and cellular component. This resulted in a total of 38 over-represented GO-categories (Fig. 2ac). $11 \mathrm{GO}$ categories corresponding to cellular component were judged as too general and non-informative (Additional file 3: Fig S2).

Interestingly, $8 \mathrm{GO}$-categories (21\%) corresponding to general transcription, and 8 GO-categories (21\%) corresponding to rRNA synthesis were over-represented in the $\mathrm{DE}$ genes list. The mitochondrial part was also overrepresented with $4 \mathrm{GO}$-categories $(11 \%)$. We isolated the gene list pertaining to the GO-categories related to general transcription, to rRNA synthesis and, using annotations from BioMart tool, all mitochondrial DE genes, including nuclear and mitochondrial encoded genes. A heatmap for each of these gene-sets was generated (Fig. 2d-f) and shows that LCL-AT have a defect in ribosome and transcription-related gene expression, and in mitochondrial genes. Gene lists related to each heatmap are given in Additional file 4: Table S2.

The rRNA fraction of the RNA prepared prior to RNAseq was analyzed by capillary gel electrophoresis. The results show that LCL-AT have a slightly lower percentage of rRNA than LCL-WT (Additional file 5: Fig. S3A). Among these rRNAs, we observed a significant decrease in the percentage of the $28 \mathrm{~S}$ RNA and $28 \mathrm{~S}+18 \mathrm{~S}$ RNA (Fig. 2g) but no difference for the 18S RNA (Additional 


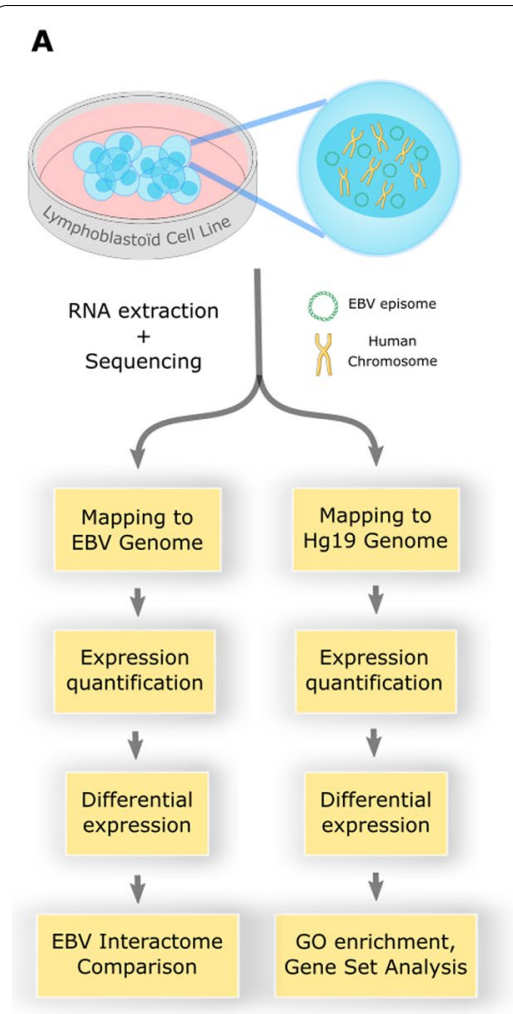

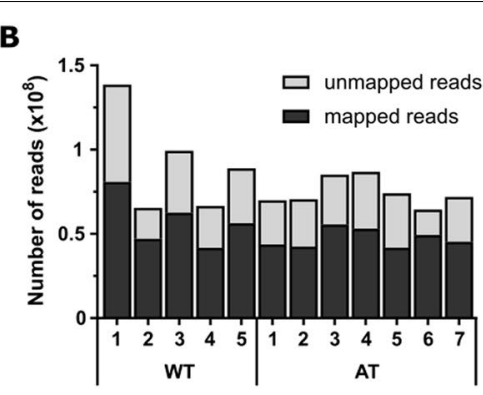

D

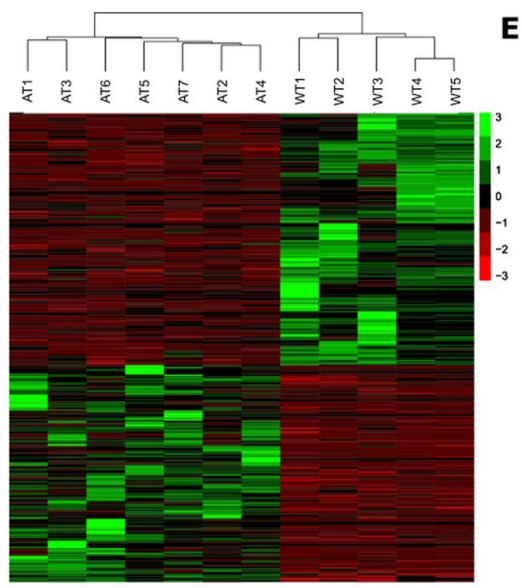

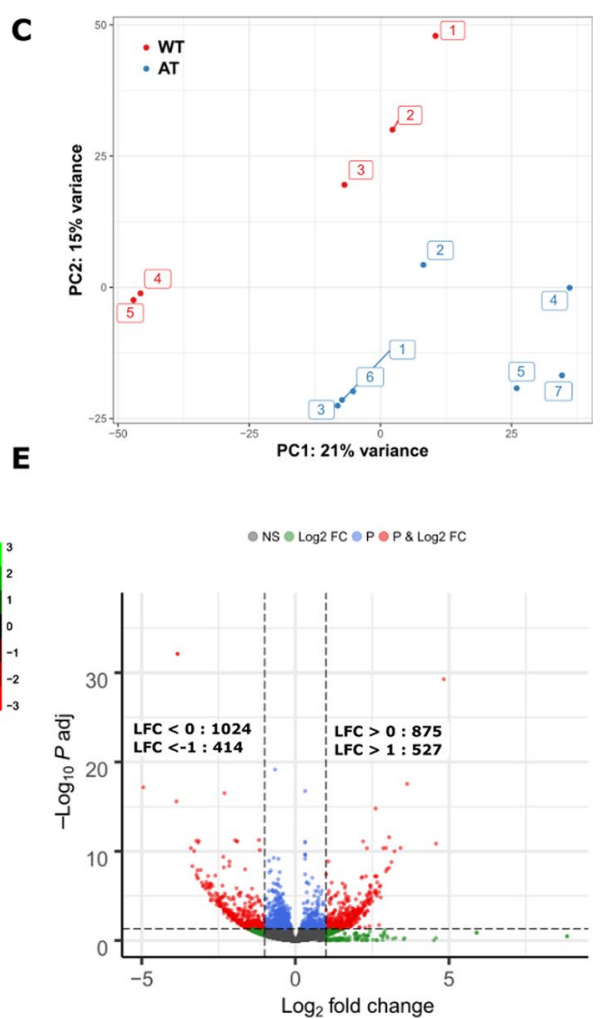

Fig. 1 Overview of RNA-seq data generated from lymphoblastoid cell lines derived from 7 AT and 5 unaffected control individuals. a Experimental design and bioinformatic data analysis for dual RNA-seq. b Result of reads alignment on the human and EBV references genomes. For each sample, the number of mapped or unmapped read are shown on the $y$-axis. c Principal component analysis (PCA) plot visualizing the similarities between biological replicates and the separation between LCL-WT (red dots) and LCL-AT (blue dots). $\mathbf{d}$ Hierarchical clustering dendrogram of LCL-WT and LCL-AT normalized gene expression, with expression levels of differentially expressed genes with $|\log 2 \mathrm{FC}|>1$ showed as a heatmap. Red represent downregulated genes and green represent upregulated genes. e Volcano plot displaying log2 fold change against $-\log 10$ ( $p$-adjusted). DESeq2 analysis identified 1024 significant downregulated genes and 875 significant upregulated genes among which 414 had a log 2 fold change $<-1$ and 527 had a log2 fold change $>1$

file 5: Fig. S3B). The $28 \mathrm{~S} / 18 \mathrm{~S}$ ratio and the RNA integrity number (RIN) attested that RNA was of good quality and that there was no difference in RNA degradation between LCL-AT and LCL-WT (Additional file 5: Fig. S3C-D).

Transcription and translation rates were assessed by incubating LCL-WT and LCL-AT with 5-ethynyl uridine (EU), an RNA nucleotide analogue, or with O-Propargylpuromycin (OPP), an agent incorporated during translation. EU incorporation did not show any significant difference in the transcription rate between LCL-WT and LCL-AT (Fig. 2h) but OPP incorporation showed a significant decrease $(p$ value $<0.05)$ in protein synthesis rate in LCL-AT (Fig. 2i). These results indicated that LCL-AT have a generalized protein synthesis defect. Mitochondrial respiration was also assessed by SeaHorse analysis but did not show any difference between LCL-WT and LCL-AT (data not shown).

In order to further analyze the DE gene pathways, we performed the Ensemble of Gene Set Enrichment
Analyses (EGSEA) [16]. Given the large amount of data generated (Additional file 6: Table S3), we kept only Gene-Sets (GS) with adjusted $p$ value $<0.05$ and absolute average $\log 2 \mathrm{FC}$ with genes regulated in the same direction as the GS (|avg.logfc.dir $\mid)>2$ (Fig. 3).

GS related to cancer were particularly enriched. DE genes were enriched in the signature of the KRASdependent Prostate Cancer in both directions. This trend was also corroborated by the GS "Neoplasm" and "Breast Cancer". However, GS related to telomere maintenance was found to be significantly downregulated in LCL-AT. DE-GS related to immunity were also particularly deregulated in LCL-AT, suggesting that LCL-AT may have immunological disorders. There was also a high enrichment of cytokines and interleukin GS in LCL-AT. Other DE-GS related to immunity included NK-cell cytotoxicity, abnormal innate immunity or abnormal level of class II molecules (Fig. 3). 


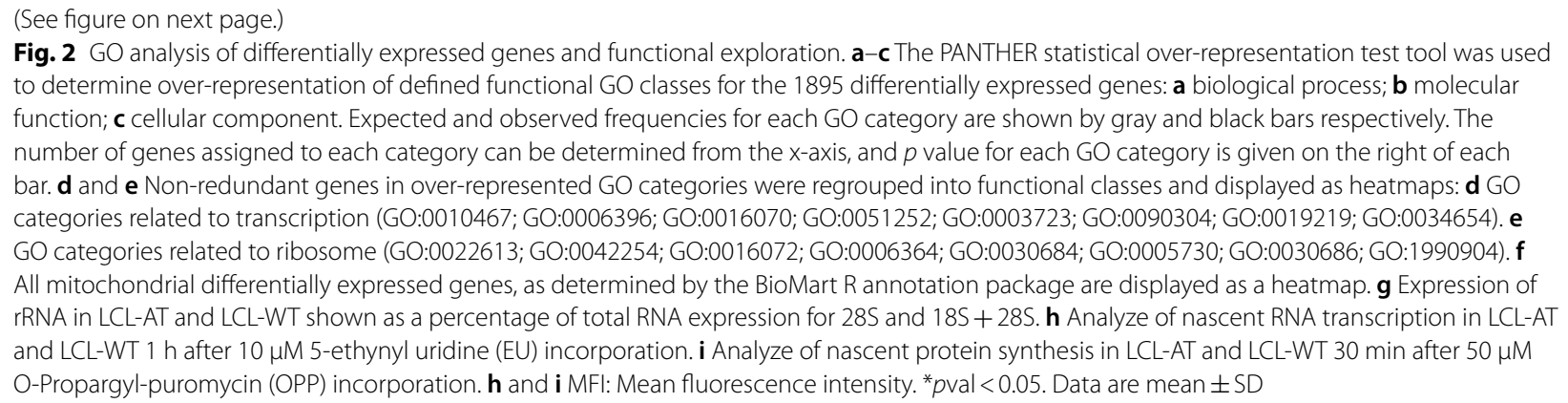

\section{Analysis of the viral gene expression}

The normalized viral counts were viewed using the IGV software (Fig. 4a). A zoom on an intergenic region showed no reads, supporting the absence of contaminating reads from cellular DNA (Additional file 7: Fig. S4). The AT2, AT4 and AT6 lines express more viral transcripts than the other lines (Fig. $4 \mathrm{~b}$ ). This could be explained by a difference in lytic cycle induction between all lines at the time of RNA extraction. Indeed, there is always $0.1-4 \%$ of cells undergoing lytic cycle [17]. EBVinfected cells in the latent phase express 9 latent genes and 2 non-coding EBERs, whereas during the lytic cycle the cells express around a hundred lytic genes under the control of BZLF1, the lytic cycle master-gene regulator [1]. Knowing that cells in latent cycle express little viral RNA, a small difference in the proportion of cells undergoing lytic cycle would be enough to drastically change the total number of viral transcripts expressed in each line. Indeed, we confirmed that the expression of BZFL1 follows the same pattern (Pearson correlation $=0.97$ ) as the total viral counts (Fig. 4c).

To verify that the small proportion of cells undergoing lytic reactivation, do not significantly modify the overall latent gene expression, we downloaded 464 EBV standardized read counts from the EBV Portal platform [18], which gather EBV RNA-seq results of LCLWT sequenced within the 1000 genome project. We established 4 quartiles according to BZLF1 expression, (Fig. 4f). Total and latent viral counts were calculated for each quartile. The results show that total viral counts depend on BZLF1 expression, with a significant increase in total viral counts between each quartile (Fig. $4 \mathrm{~g}$ ), but not latent viral counts (Fig. 4h). Total viral counts are relative to BZLF1 expression and have no correlation with latent counts. This allows us to analyze latent genes, regardless of lytic genes expression.

Differential analysis of latent genes did not show any DE genes between LCL-AT and LCL-WT (Fig. 4d, e) and all latent genes were expressed in LCL-WT and LCLAT. We compared the cellular DE genes list with the non-redundant viral latent genes interactome available on the Polygenic pathways database. From this analysis we found 71 interactions representing $54 \mathrm{DE}$ genes, of which 42 have an $|\log 2 \mathrm{FC}|>1$. EBNA-3A appears to interact with 39 DE genes (Fig. 5a), EBNA-3C with 11 DE genes (Fig. 5b) and LMP1 with 11 DE genes (Fig. 5c). These data suggest that although the EBNA-3A, EBNA-3C and LMP1 transcripts were not DE, the activity of these genes may be disrupted in LCL-AT. Interestingly, most of the DE genes described as downregulated or upregulated by EBNA-3A were also downregulated or upregulated in LCL-AT respectively, indicating that EBNA-3A may be more active in LCL-AT than in LCL-WT.

\section{Discussion}

AT patients have a high risk of developing lymphoid malignancies with a high rate of association with EBV. Mechanisms associated with this specific susceptibility may be due cellular immune deficiency in a number of AT patients, or to other specific immune dysfunctions that remain to be explored. We raised the hypothesis that ATM defect in EBV-infected cells could play a role per se in the control of EBV latency, favoring a latent program more prone to lymphomagenesis [19]. In the present study, we used strand-specific RNA seq strategy to profile the RNA expression landscape of ATM deficient LCL versus control, in order to assess the involvement of ATM in EBV latent cycle regulation.

Our data suggests a previously unsuspected ribosomal defect in LCL-AT. In addition, we found that LCLAT display a distinct pattern of cancer associated gene expression, most notably by overexpressing certain oncogenes and downregulation of tumor suppressors, and also exhibit features of immune dysfunction. We also confirmed that latent gene expression can be studied regardless of lytic gene expression. Our data, based on the single technique of RNA-seq analysis, will require validation by additional studies. 


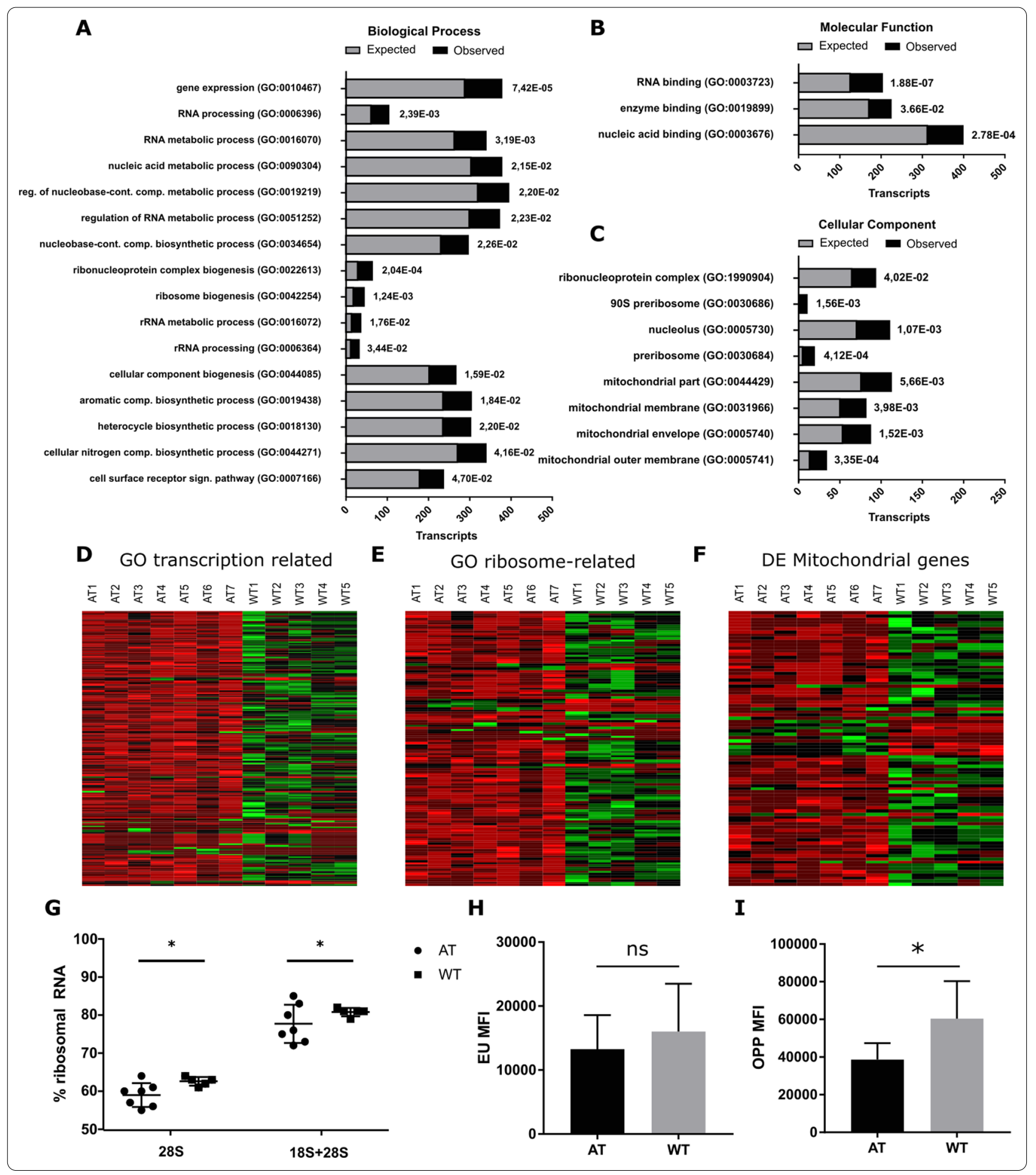

\section{Transcription, translation and mitochondria}

Our results suggest that LCL-AT may have a transcriptional and translational defect. Indeed LCL-AT express less 28S RNA and have a lower translational rate than LCL-WT, but transcription capacities did not differ significantly from LCL-WT. The possible transcriptional defect does not appear to affect EBV latency genes, as observed by the absence of DE latency genes between LCL-AT and LCL-WT. Housekeeping genes were not $\mathrm{DE}$, suggesting that transcriptional alterations may affect 


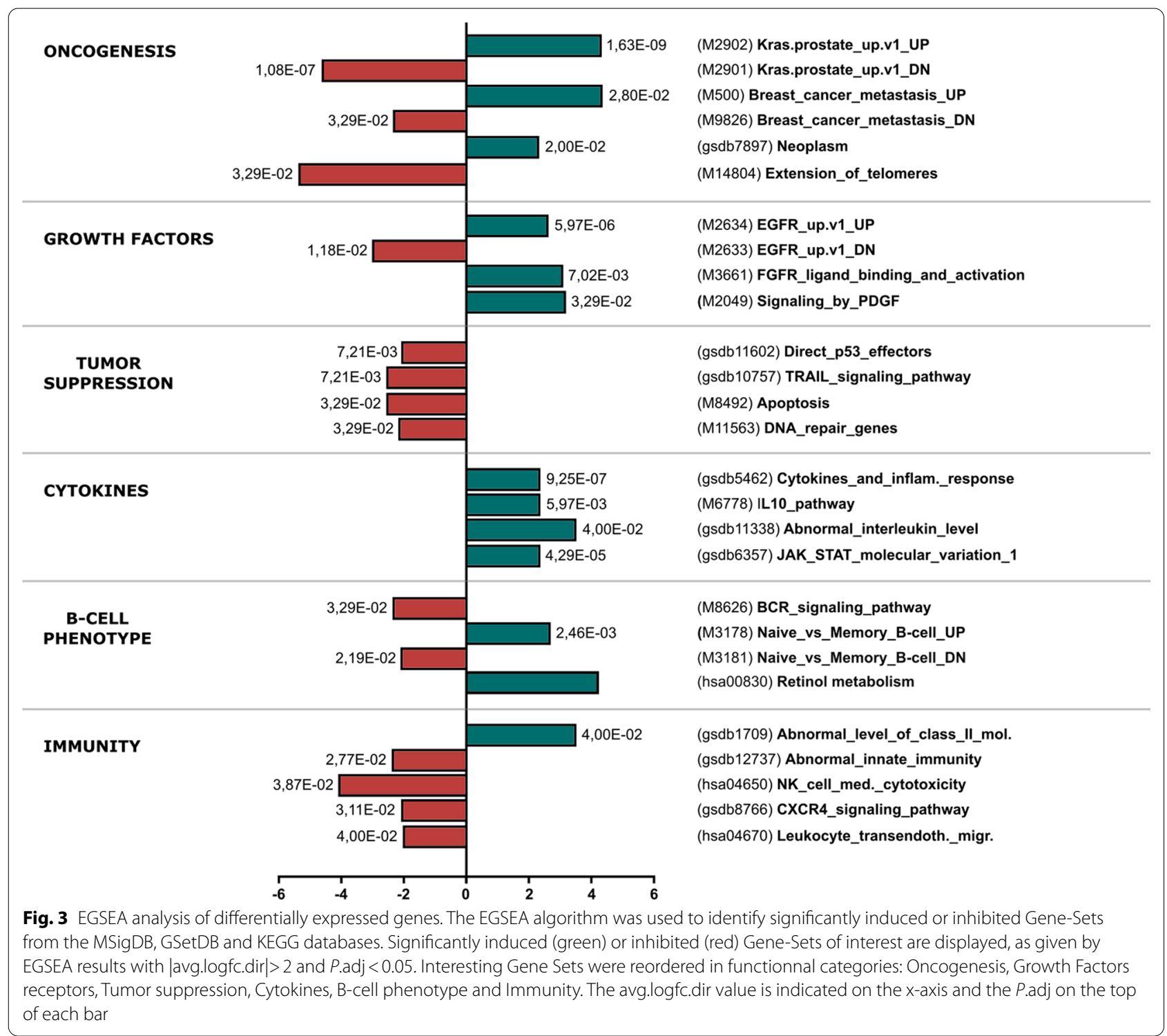

specific genes such as ribosomal genes. Interestingly, the signaling pathways for transcription and translation were not found in the EGSEA results but stand out significantly with a lower log.fold.dir. This underscores the need for several complementary methods to study RNAseq data.

The alteration of 28S RNA and translational rate in LCL-AT suggest that the pathophysiology of ataxia-telangiectasia may also include aspects of ribosomal disease. ATM participates in the modification of the nucleolus architecture in case of double-strand break within rDNA [20] and It has been suggested that ATM participates in basal nucleolar transcription [21]. Other immunodeficiencies with specific susceptibility to EBV-such as CTPS1 deficiency-are characterized by altered nucleic acid metabolism leading to rapid T-cell exhaustion upon massive proliferation induced by EBV infection [22]. We hypothesize that the massive protein synthesis rate in cytotoxic T-cells during EBV-driven proliferative stress is inefficiently sustained in ataxia-telangiectasia, resulting in a defective control of EBV. Further studies are needed to address this hypothesis. Transcription of many mitochondrial genes were decreased in LCL-AT including several genes involved in the respiratory chain and in ribosomal protein synthesis. Inhibition of ATM leads to mitochondrial dysfunction and ROS production [23]. The latter could be involved in the increased incidence of cancers in patients with ataxia-telangiectasia by increasing genotoxic stress. 


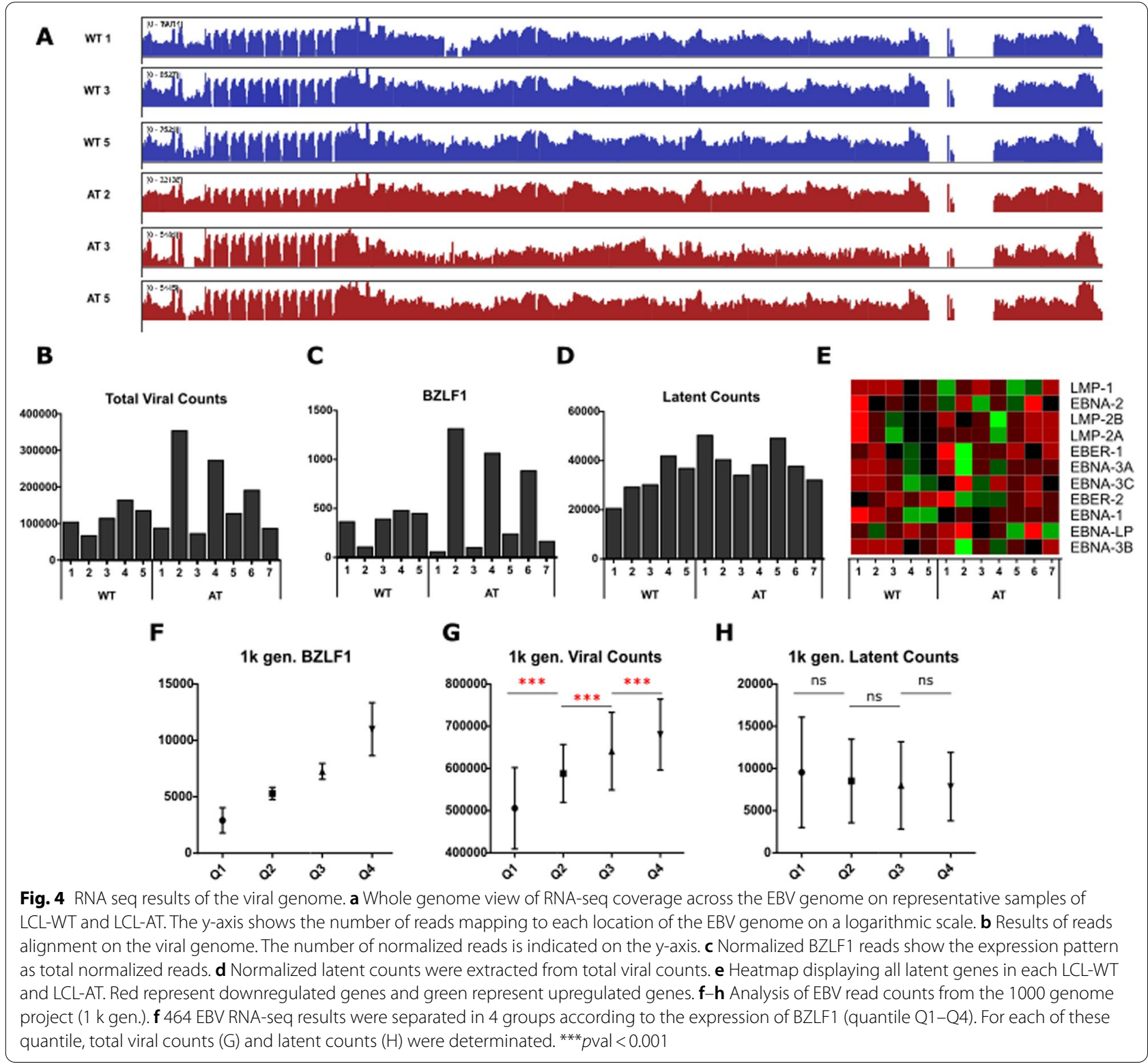

\section{Oncogenesis and immune dysfunction}

The EGSEA results show enrichment in pro-tumorigenic GS particularly oncogenes, growth factors and downregulation of tumor suppressors in keeping with the increased cancer risk in AT [24]. Among the main oncogenes induced in LCL-AT, we find BCL11A $(\log 2 \mathrm{FC}$ 4.20), a modulator of transcriptional repression frequently upregulated in B-cell malignancies $[25,26]$ or TCL1A $(\log 2 \mathrm{FC} 3.41)$, a survival promoting factor strongly associated with Burkitt lymphoma and related to other malignancies $[27,28]$. The main tumor suppressors downregulated in AT are PCDH10 $(\log 2 \mathrm{FC}-4.76)$, a protocadherin whose promoter is methylated in diffuse large B-cell lymphomas [29] or PTPN13 (log FC: -2.84) an inhibitor of FAS-induced apoptosis associated with aggressive breast cancer [30].

Telomere maintenance pathway, including TERT, was downregulated in LCL-AT, $(\log 2 \mathrm{FC}-4.78)$. Inhibition of TERT in LCL decreases cell proliferation and induces apoptosis in an ATM dependent manner [31] as well as induces the EBV lytic cycle, which was not the case in LCL-AT (data not shown). LCL-AT may use an alternative lengthening of telomeres pathway [32]. Whether TERT related induction of the EBV lytic cycle is also ATM dependent should be further explored. 


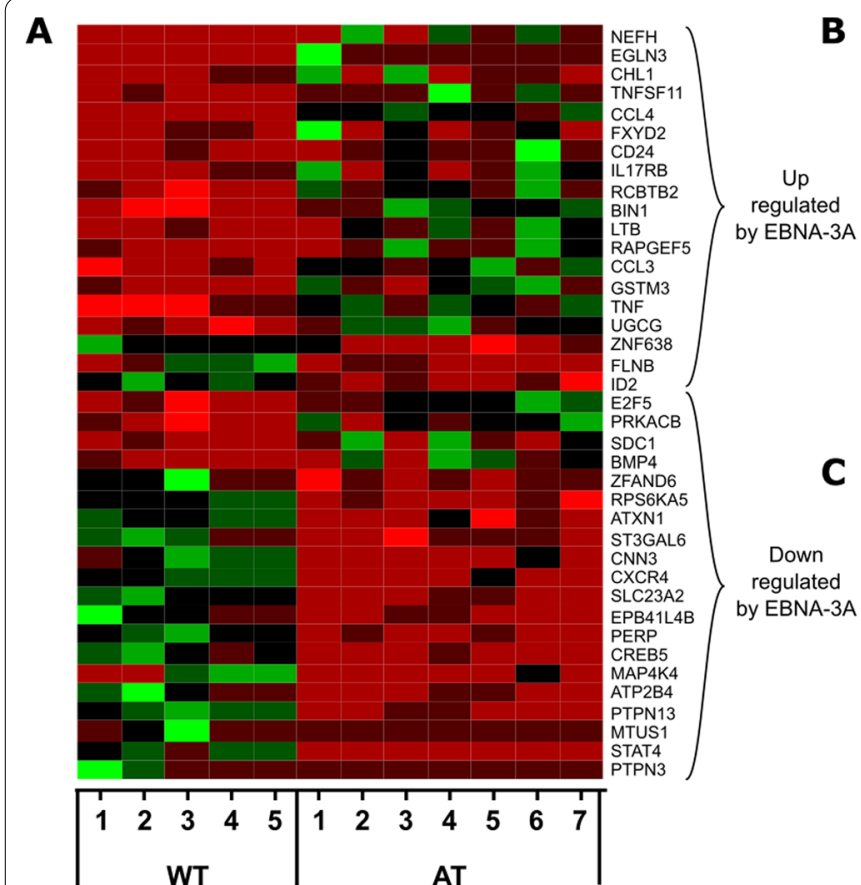

B

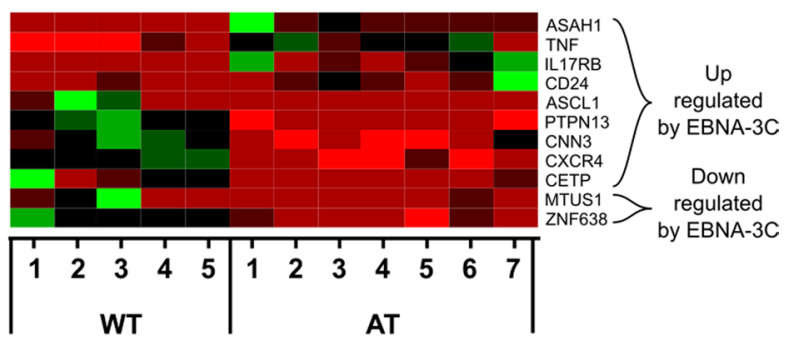

\section{C}
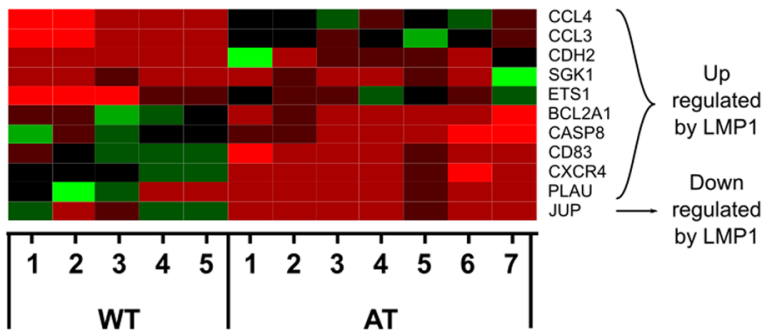
by LMP1

Fig. 5 Heatmaps of EBV interactome. a-c Heatmaps showing all differentially expressed genes that were described, according to the Polygenicpathway EBV database, as regulated by: a EBNA-3A, b EBNA-3C, c LMP1

A modulation of innate immunity in LCL-AT is suggested by several DE-GS. The gene expression of HLA$C$, a major NK cell inhibitory molecule, is upregulated in LCL-AT ( $\log 2 \mathrm{FC} 5.63)$. Similarly, CD200R1, CD276, SLAMF7, LILRB1 were overexpressed, suggesting that AT patients may have a disrupted NK cell function. On the other hand, LAIR1, another inhibitory molecule, was downregulated $(\log 2 \mathrm{FC}-4.99)$.

IL4 and IL10 were also upregulated in LCL-AT ( $\log 2 \mathrm{FC} 2.23$ and 3.58 , respectively). These two cytokines participate in the proliferation, plasma cell differentiation and antibody production of $\mathrm{B}$ lymphocytes [33, 34]. IL10 also inhibits CD8 cytotoxic T-cells [35]. The cGAS, STING (TMEM173) [36] and interferon $\beta 1$ (IFNB1) transcripts were downregulated $(\log 2 \mathrm{FC}-0.70 ;-2.39$ and -2.51 respectively), suggesting a possible defect in antiviral response in LCL-AT.

\section{EBV regulation}

We found no significant difference in EBV latent gene expression between LCL-AT and LCL-WT. Several deregulated cellular genes in LCL-AT interact with EBNA-3A, EBNA-3C and LMP1 suggesting an overall differential impact on cellular homeostasis.
ATM participates in the regulation of EBV's lytic cycle and is necessary for a proper viral replication in epithelial cells [37]. In LCL however, ATM inhibition through caffeine treatment [38] or the lack of ATM in our LCLAT did not affect viral replication. On the other hand, LCL treated by the pan-PIKK inhibitor (to which the ATM kinase belongs) LY294002 were shown to inhibit viral replication. It is thus possible that another kinase compensates for ATM deficiency in LCL, to promote viral replication. ATR is a good candidate as it activates the same downstream targets as ATM.

However, the impact of ATM on the control of EBV latency may not be manifested in a highly artificial system such as LCL, but appear in the context of natural EBV-B cell infection.

\section{Conclusion}

In summary, we show that LCL-AT display a gene expression pattern consistent with the observed increased incidence of EBV-related malignancies in patients with Ataxia-Telangiectasia. The dysregulated pathways uncovered by this approach need to be further explored to better understand the biological mechanisms involved in the regulation of EBV latency and lymphomagenesis. Elucidation of these pathways may contribute to the development of novel approaches to 
treat or prevent EBV associated lymphoproliferations in AT patients where conventional chemotherapy is very toxic because of the DDR defect, and also in the general population.

\section{Methods}

\section{Cell lines and culture}

LCL were generated according to standard protocols at the Genethon Laboratory and at the Imagine Institute Biological Resource Center. LCL were cultured in RPMI containing $10 \% \mathrm{FBS}$ at $37{ }^{\circ} \mathrm{C}$ in a $5 \% \mathrm{CO}_{2}$ incubator and passed every 3 days replacing half of the culture medium with fresh medium.

\section{RNA extraction and sequencing}

LCL-WT and LCL-AT were harvested in an exponential growth phase. Total RNA was extracted using the RNeasy Plus Mini Kit (Qiagen). The concentration of total RNA was measured spectrometrically using Xpose (Trinean). The RNA integrity was analyzed by capillary electrophoresis using a Tape-Station (Agilent). The NUGEN Ovation Universal RNA-Seq system was used to construct the RNA-seq libraries from $100 \mathrm{ng}$ of total RNA according to the manufacturer protocol. RNA sequencing was performed by the genomics platform of Imagine Institute on HiSeq 2500 (Illumina), by multiplexing 12 libraries per line to obtain a sequencing depth of 70 million pair-end reads per library, with a read length of approximately 130 nucleotides.

\section{Reads quantification and differential analysis}

Salmon v0.8.2 [39] was used to pseudo-align raw RNAseq reads to both human and viral genomes and get quantification estimates at the transcript level. The human reference genome GRCh38 was downloaded from the ENSEMBL website [40] (http://ftp.ensembl. org/pub/release-92/fasta/homo_sapiens/cdna), and the EBV genome B95-8/Raji from Flemington Lab (http:// www.flemingtonlab.com/rnaseq.html). Differential analysis was performed using $\mathrm{R}$ software, version 3.5.0 [41] and the negative binomial generalized linear modelling implemented in DESeq2 package version 1.20.0 [42]. A Wald test was applied on viral transcripts to perform comparisons among conditions. $p$ Values were corrected for multiple testing using Benjamini-Hochberg correction [43]. The BioMart R package [44] was used to annotate human differentially expressed genes.

\section{Genome visualization}

Reads were mapped on the human genome (hg38) using STAR v2.5.0a [45] and the unmapped reads were aligned to the EBV genome using bowtie2 v2.3.4.2 [46]. The format conversions, sorting, and indexing intermediate operations on the data were performed using samtools v1.7 [47] and bedtools v2.26 [48]. The snapshots obtained in Fig. 4a and Additional file 7: Fig S4 were obtained with IGV v2.4.15 [49].

\section{Gene ontology and gene sets analysis}

Differentially expressed genes were subjected to GOAnalysis, using the over-representation test within the PANTHER classification system [50] (version 13.1). Statistically over-represented GO-categories $(\mathrm{FDR}<0.05)$ containing less than $25 \%$ of the input were selected for visualization. Functional analysis was carried out using the Ensemble of Gene Set Enrichment Analyses (EGSEA) $\mathrm{R}$ package (version 1.8.0). EGSEA uses 12 algorithms and combine the results by calculating a Wilkinson adjusted p-value. The EGSEA database consists of approximately 25.000 genes-sets classified into 16 collections from the MSigDB [51, 52], GeneSetDB [53] and KEGG [54] databases.

\section{Transcription and translation assay}

Cells were incubated with 5-ethynyl uridine (EU) on $1 \mathrm{~h}$ or with O-Propargyl-puromycin (OPP) on $30 \mathrm{~min}$. Transcription and translation rate were assessed using the Click-it assay Kit (Invitrogen), followed by flow-cytometer analysis. Statistical comparison between LCL-AT and LCL-WT were performed using a Mann-Whitney-Wilcoxon non-parametric test, using Prism (version 7.00, GraphPad Software).

\section{Genome project and EBV interactome data}

EBV standardized read counts sequenced within the 1000 genome project were downloaded on the EBV Portal platform (https://ebv.wistar.upenn.edu) and the EBV interactome data from the Polygenic pathways website (http://www.polygenicpathways.co.uk/epsteinbarr.htm).

\section{Abbreviations \\ AT: ataxia-telangiectasia; |avg.logfc.dir|: average log2FC with genes regulated in the same direction as the GS; DDR: DNA damage response; DE: differentially expressed; EBV: Epstein-Barr virus; EGSEA: ensemble of gene set enrichment analyses; EU: 5-ethynyl uridine; GS: gene-set; LCL: lymphoblastoid cell line; |log2FC|: absolute Log2 fold-change; OPP: O-propargyl-puromycin; PANTHER: protein analysis through evolutionary relationships; PID: primary immune deficiencies; RIN: RNA integrity number.}

\section{Supplementary Information}

The online version contains supplementary material available at https://doi. org/10.1186/s13023-021-01904-3.

Additional file 1: Figure S1. ATM expression and exploration of its function. 
Additional file 2: Table S1 List of 1899 differentially expressed genes. Additional file 3: Figure S2. GO analysis of differentially expressed genes. Additional file 4: Table S2 List of Mitochondrial, RNA-related and ribosome-related differentially expressed genes.

Additional file 5: Figure S3. Exploration of rRNA content.

Additional file 6: Table S3. EGSEA results for differentially expressed genes.

Additional file 7: Figure S4. RNA-seq coverage across an intergenic region of EBV genome.

\section{Acknowledgements}

The authors would like to thank Pr Marc Lecuit, Institut Pasteur, for helpful discussions.

\section{Authors' contributions}

MT contributed to the content and drafted the manuscript. EP, KHH, MAD and HM analyzed and interpreted the data. OH was responsible for critical revision of the manuscript. FS supervised the study and contributed to the writing of the manuscript. All authors read and approved the final manuscript.

\section{Funding}

This work was supported in part by grants from Fondation ARC pour la recherche sur le cancer (France), Fondation ATEurope (France) and Le Centre de Référence Déficits Immunitaires Héréditaires (CEREDIH, France).

\section{Availability of data and materials}

All data generated or analyzed during the current study are included in this published article.

\section{Declarations}

\section{Ethics approval and consent to participate}

Not applicable.

\section{Consent for publication}

Not applicable.

\section{Competing interests}

The authors declare no conflicts of interest.

\section{Author details}

'INSERM U1163/CNRS ERL8254 - Laboratory of Cellular and Molecular Mechanisms of Hematological Disorders and Therapeutic Implications, Institut Imagine, Paris, France. ${ }^{2}$ Department of Adult Hematology, AP-HP. Centre, Necker - Enfants Malades Hospital, Université de Paris, Paris, France. ${ }^{3}$ Université de Paris, Paris, France. ${ }^{4} \mathrm{Hub}$ de Bioinformatique et Biostatistique - Département Biologie Computationnelle, Institut Pasteur, Paris, France.

\section{Received: 29 January 2021 Accepted: 7 June 2021}

Published online: 28 June 2021

\section{References}

1. Young LS, Rickinson AB. Epstein-Barr virus: 40 years on. Nat Rev Cancer. 2004:4(10):757-68.

2. Miyashita EM, Yang B, Lam KM, Crawford DH, Thorley-Lawson DA. A novel form of Epstein-Barr virus latency in normal $B$ cells in vivo. Cell. 1995;80(4):593-601.

3. Cohen JI. Primary immunodeficiencies associated with EBV disease. Curr Top Microbiol Immunol. 2015:390(Pt 1):241-65.

4. Suarez F, Mahlaoui N, Canioni D, Andriamanga C, Dubois d'Enghien C, Brousse N, et al. Incidence, presentation, and prognosis of malignancies in ataxia-telangiectasia: a report from the French national registry of primary immune deficiencies. J Clin Oncol Off J Am Soc Clin Oncol. 2015;33(2):202-8
5. Lavin MF. Ataxia-telangiectasia: from a rare disorder to a paradigm for cell signalling and cancer. Nat Rev Mol Cell Biol. 2008;9(10):759-69.

6. Nowak-Wegrzyn A, Crawford TO, Winkelstein JA, Carson KA, Lederman HM. Immunodeficiency and infections in ataxia-telangiectasia. J Pediatr. 2004:144(4):505-11.

7. Latour S, Winter S. inherited immunodeficiencies with high predisposition to Epstein-Barr virus-driven lymphoproliferative diseases. Front Immunol. 2018;9:1103.

8. Shiloh Y, Ziv Y. The ATM protein kinase: regulating the cellular response to genotoxic stress, and more. Nat Rev Mol Cell Biol. 2013;14(4):197-210.

9. Matsuoka S, Ballif BA, Smogorzewska A, McDonald ER, Hurov KE, Luo $J$, et al. ATM and ATR substrate analysis reveals extensive protein networks responsive to DNA damage. Science. 2007:316(5828):1 160-6.

10. Shanbhag NM, Rafalska-Metcalf IU, Balane-Bolivar C, Janicki SM, Greenberg RA. ATM-dependent chromatin changes silence transcription in cis to DNA double-strand breaks. Cell. 2010;141(6):970-81.

11. Harding SM, Boiarsky JA, Greenberg RA. ATM dependent silencing links nucleolar chromatin reorganization to DNA damage recognition. Cell Rep. 2015:13(2):251-9.

12. Kulinski JM, Leonardo SM, Mounce BC, Malherbe L, Gauld SB, Tarakanova VL. Ataxia telangiectasia mutated kinase controls chronic gammaherpesvirus infection. J Virol. 2012;86(23):12826-37.

13. Hollingworth R, Skalka GL, Stewart GS, Hislop AD, Blackbourn DJ, Grand RJ. Activation of DNA damage response pathways during lytic replication of KSHV. Viruses. 2015;7(6):2908-27.

14. Mi H, Huang X, Muruganujan A, Tang H, Mills $C$, Kang D, et al. PANTHER version 11: expanded annotation data from gene ontology and reactome pathways, and data analysis tool enhancements. Nucleic Acids Res. 2017;45(D1):D183-9.

15. Beissbarth T. Speed TP. GOstat: find statistically overrepresented gene ontologies within a group of genes. Bioinform Oxf Engl. 2004;20(9):1464-5

16. Alhamdoosh M, Law CW, Tian L, Sheridan JM, Ng M, Ritchie ME. Easy and efficient ensemble gene set testing with EGSEA. F1000Research. 2017:6:2010.

17. Vrzalikova K, Vockerodt M, Leonard S, Bell A, Wei W, Schrader A, et al. Down-regulation of BLIMP1a by the EBV oncogene, LMP-1, disrupts the plasma cell differentiation program and prevents viral replication in B cells: implications for the pathogenesis of EBV-associated B-cell lymphomas. Blood. 2011;117(22):5907-17.

18. Arvey A, Tempera I, Tsai K, Chen H-S, Tikhmyanova N, Klichinsky M, et al. An atlas of the Epstein-Barr virus transcriptome and epigenome reveals host-virus regulatory interactions. Cell Host Microbe. 2012;12(2):233-45.

19. Tatfi M, Hermine O, Suarez F. Epstein-Barr virus (EBV)-related lymphoproliferative disorders in ataxia telangiectasia: Does ATM regulate EBV life cycle? Front Immunol. 2018;9:3060.

20. Larsen DH, Stucki M. Nucleolar responses to DNA double-strand breaks. Nucleic Acids Res. 2016;44(2):538-44.

21. Smith SC, Hetman M. ATM is a novel regulator of nucleolar transcription. FASEB J. 2011;25(1):Ib34.

22. Martin E, Noé P, Sylvia S, Christelle L, Fabian H, Cédric M, Sylvie F, et al. CTP synthase 1 deficiency in humans reveals its central role in lymphocyte proliferation. Nature. 2014;510(74):288-92.

23. Reichenbach J, Schubert R, Schindler D, Müller K, Böhles H, Zielen S Elevated oxidative stress in patients with ataxia telangiectasia. Antioxid Redox Signal. 2002;4(3):465-9.

24. Suarez F, Mahlaoui N, Canioni D, Andriamanga C, d'Enghien CD, Brousse $\mathrm{N}$, et al. Incidence, presentation, and prognosis of malignancies in ataxiatelangiectasia: a report from the French national registry of primary immune deficiencies. J Clin Oncol. 2015:33(2):202-8.

25. Martín-Subero Jl, Gesk S, Harder L, Sonoki T, Tucker PW, Schlegelberger $B$, et al. Recurrent involvement of the REL and BCL11A loci in classical Hodgkin lymphoma. Blood. 2002;99(4):1474-7.

26. Weniger MA, Pulford K, Gesk S, Ehrlich S, Banham AH, Lyne L, et al. Gains of the proto-oncogene BCL11A and nuclear accumulation of $B C L 11 \mathrm{~A}(X \mathrm{~L})$ protein are frequent in primary mediastinal B-cell lymphoma. Leukemia. 2006;20(10):1880-2

27. Aggarwal M, Villuendas R, Gomez G, Rodriguez-Pinilla SM, Sanchez-Beato M, Alvarez D, et al. TCL1A expression delineates biological and clinical 
variability in B-cell lymphoma. Mod Pathol Off J U S Can Acad Pathol. 2009;22(2):206-15.

28. Gaudio E, Paduano F, Pinton S, D'Agostino S, Rocca R, Costa G, et al. TCL1A interacts with TP63 and enhances the survival of Raji Burkitt lymphoma cell line. Br J Haematol. 2018;183(3):509-12.

29. Huang W, Xue X, Shan L, Qiu T, Guo L, Ying J, et al. Clinical significance of PCDH10 promoter methylation in diffuse large B-cell lymphoma. BMC Cancer. 2017;17(1):815.

30. Glondu-Lassis M, Dromard M, Lacroix-Triki M, Nirdé P, Puech C, Knani D, et al. PTPL1/PTPN13 regulates breast cancer cell aggressiveness through direct inactivation of Src kinase. Cancer Res. 2010;70(12):5116-26.

31. Celeghin A, Giunco S, Freguja R, Zangrossi M, Nalio S, Dolcetti R, et al. Short-term inhibition of TERT induces telomere length-independent cell cycle arrest and apoptotic response in EBV-immortalized and transformed B cells. Cell Death Dis. 2016;7(12):e2562.

32. Cesare AJ, Reddel RR. Alternative Lengthening of Telomeres in Mammalian Cells [Internet]. Landes Bioscience; 2013 [cité 31 déc 2018]. Disponible sur: https://www.ncbi.nlm.nih.gov/books/NBK6486/

33. Moens L, Tangye SG. Cytokine-mediated regulation of plasma cell generation: IL-21 takes center stage. Front Immunol. 2014;5:65.

34. Bod L, Douguet L, Auffray C, Lengagne R, Bekkat F, Rondeau E, et al. |L-4-induced gene 1: a negative immune checkpoint controlling B cell differentiation and activation. J Immunol Baltim Md 1950. 2018;200(3):1027-38.

35. Sun Z, Fourcade J, Pagliano O, Chauvin J-M, Sander C, Kirkwood JM, et al. IL10 and PD-1 cooperate to limit the activity of tumor-specific CD8+ T cells. Cancer Res. 2015;75(8):1635-44.

36. Härtlova A, Erttmann SF, Raffi FAM, Schmalz AM, Resch U, Anugula S, et al. DNA damage primes the type I Interferon system via the cytosolic DNA sensor STING to promote anti-microbial innate immunity. Immunity. 2015;42(2):332-43.

37. Hau PM, Deng W, Jia L, Yang J, Tsurumi T, Chiang AKS, et al. Role of ATM in the formation of the replication compartment during lytic replication of Epstein-Barr virus in nasopharyngeal epithelial cells. J Virol. 2015;89(1):652-68.

38. Kudoh A, Fujita M, Zhang L, Shirata N, Daikoku T, Sugaya Y, et al. EpsteinBarr virus lytic replication elicits ATM checkpoint signal transduction while providing an S-phase-like cellular environment. J Biol Chem. 2005;280(9):8156-63.

39. Patro R, Duggal G, Love M, Irizarry R, Kingsford C. Salmon provides fast and bias-aware quantification of transcript expression. Nat Methods. 2017:14(4):417-9.

40. Zerbino DR, Achuthan P, Akanni W, Amode MR, Barrell D, Bhai J, et al. Ensembl 2018. Nucleic Acids Res. 2018;46(D1):D754-61.
41. R Core Team. R: A language and environment for statistical computing. R Foundation for Statistical Computing, Vienna, Austria. 2018. URL https:// www.R-project.org/.

42. Love MI, Huber W, Anders S. Moderated estimation of fold change and dispersion for RNA-seq data with DESeq2. Genome Biol. 2014;15(12):550.

43. Benjamini $Y$, Hochberg $Y$. Controlling the false discovery rate: a practical and powerful approach to multiple testing. J R Stat Soc Ser B Methodol. 1995;57(1):289-300.

44. Durinck S, Spellman PT, Birney E, Huber W. Mapping identifiers for the integration of genomic datasets with the R/Bloconductor package biomaRt. Nat Protoc. 2009;4(8):1184-91.

45. Dobin A, Davis CA, Schlesinger F, Drenkow J, Zaleski C, Jha S, et al. STAR: ultrafast universal RNA-seq aligner. Bioinform Oxf Engl. 2013;29(1):15-21.

46. Langmead B, Salzberg SL. Fast gapped-read alignment with Bowtie 2. Nat Methods. 2012;9(4):357-9.

47. Li H, Handsaker B, Wysoker A, Fennell T, Ruan J, Homer N, et al. The sequence alignment/map format and SAMtools. Bioinform Oxf Engl. 2009;25(16):2078-9.

48. Quinlan AR, Hall IM. BEDTools: a flexible suite of utilities for comparing genomic features. Bioinform Oxf Engl. 2010;26(6):841-2.

49. Thorvaldsdottir H, Robinson JT, Mesirov JP. Integrative Genomics Viewer (IGV): high-performance genomics data visualization and exploration. Brief Bioinform. 2013;14(2):178-92.

50. Mi H, Muruganujan A, Casagrande JT, Thomas PD. Large-scale gene function analysis with the PANTHER classification system. Nat Protoc. 2013;8(8):1551-66.

51. Subramanian A, Tamayo P, Mootha VK, Mukherjee S, Ebert BL, Gillette MA, et al. Gene set enrichment analysis: a knowledge-based approach for interpreting genome-wide expression profiles. Proc Natl Acad Sci USA. 2005;102(43):15545-50.

52. Liberzon A, Birger C, Thorvaldsdóttir H, Ghandi M, Mesirov JP, Tamayo P. The molecular signatures database (MSigDB) hallmark gene set collection. Cell Syst. 2015;1 (6):417-25.

53. Araki H, Knapp C, Tsai P, Print C. GeneSetDB: a comprehensive meta-database, statistical and visualisation framework for gene set analysis. FEBS Open Bio. 2012;2:76-82.

54. Kanehisa M, Goto S. KEGG: kyoto encyclopedia of genes and genomes. Nucleic Acids Res. 2000;28(1):27-30.

\section{Publisher's Note}

Springer Nature remains neutral with regard to jurisdictional claims in published maps and institutional affiliations.
Ready to submit your research? Choose BMC and benefit from:

- fast, convenient online submission

- thorough peer review by experienced researchers in your field

- rapid publication on acceptance

- support for research data, including large and complex data types

- gold Open Access which fosters wider collaboration and increased citations

- maximum visibility for your research: over $100 \mathrm{M}$ website views per year

At BMC, research is always in progress.

Learn more biomedcentral.com/submissions 